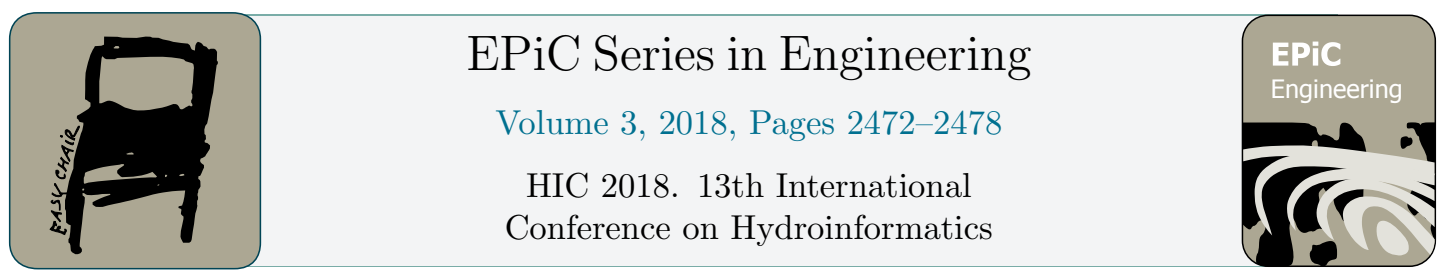

\title{
A grid convergence study for the integral porosity shallow water model on unstructured triangular meshes
}

\author{
Ilhan Özgen-Xian ${ }^{12}$, Dongfang Liang ${ }^{3}$, and Reinhard Hinkelmann ${ }^{1}$ \\ 1 Technische Universität Berlin, Berlin, Germany \\ ilhan.oezgen@wahyd.tu-berlin.de \\ 2 RIKEN Advanced Institute for Computational Science, Kōbe, Hyōgo, Japan \\ ${ }^{3}$ University of Cambridge, Cambridge, U.K.
}

\begin{abstract}
The integral porosity shallow water model (IP) is a modified form of the depth-averaged shallow water flow model, which utilizes porosities to account for unresolved sub-grid-scale topography such as buildings to enable fast urban flooding simulations. Existing research has repeatedly pointed out that the IP model is inherently oversensitive to the mesh design. This paper presents a detailed grid convergence study of the IP model for simulating a laboratory experiment on the interaction between a dam-break wave and an obstacle in a channel, which is featured by the highly complex non-hydrostatic flow with a backwardspropagating hydraulic jump. We compare three different mesh refinement techniques with up to six levels of refinement: (1) uniform, (2) manual, (3) locally coarsened. For this investigated case, the modeling error due to the shallow water assumptions is more significant than that due to the porosity treatment. Neither a conventional shallow water model, nor the integral porosity model is able to predict the measured data well owning to non-hydrostatic flow conditions and a backwards propagating hydraulic jump. We show that the integral porosity model results converge to the conventional shallow water model results at locations that are not affected by these non-hydrostatic flow conditions. We conclude that, when the obstacle density is low, high-frequency oscillations may appear in the domain owing to Kármán vortex shedding. These cannot be captured accurately by the integral porosity shallow water model, unless high resolutions similar to those in the conventional shallow water models are used. However, the benefit of the porosity model is lost by using high resolutions.
\end{abstract}

\section{Introduction}

Porous shallow water equations describe free surface shallow flow at the macroscale, i.e. obstacles are typically smaller than the control volume size, in order to reduce the computational cost. Initially derived in [3], early porous shallow water models used a representative elementary area (REA) assumption to derive a single porosity parameter to limit both storage and flux terms $[8,7]$. It was quickly discovered that a single porosity parameter is not sufficient to accurately account for preferential flow paths. Hence, in [9], an integral formulation of shallow water equations with anisotropic porosity was proposed. This model is referred to as the integral

G. La Loggia, G. Freni, V. Puleo and M. De Marchis (eds.), HIC 2018 (EPiC Series in Engineering, vol. 3), 
porosity shallow water model (IP). Using an integral formulation removes the necessity for a REA assumption and allows to use additional porosity parameters (conveyance porosity terms) at the boundaries of the control volume, i.e. the cell edges in a mesh-based discretization of the equations. The IP model results are very mesh-dependent. In [9], it is suggested to use so-called gap-conforming meshes to ensure a reliable calculation of the conveyance porosity terms. In this type of meshes, cell vertices are forced to coincide with the centroids of the obstacles. The question we aim to answer in this study is, whether it is possible to prove grid convergence for such a mesh-dependent model. Here, an additional challenge is that grid convergence should be obtained at the macroscale, i.e. cell sizes should be larger than the average obstacle size. In this work, we study the mesh-dependency of the IP model in [9]. However, it is noted that in recent years other porous shallow water models have been derived in, e.g. $[6,5,14,15,1]$. As a rule of thumb, models that use the REA assumption do not tend to be as mesh-dependent as the IP model.

\section{Governing equations}

The governing equations of the IP model are written as

$$
\int_{\Omega} \partial_{t} \phi_{\Omega} \mathbf{q} d \Omega+\oint_{\Gamma} \phi_{\Gamma} \mathbf{F} \cdot \mathbf{n} d \Gamma=\int_{\Omega} \mathbf{s}_{\Omega} d \Omega+\oint_{\Gamma} \mathbf{s}_{\Gamma} d \Gamma
$$

where $\Omega$ represents the area of the cell, $\phi_{\Omega}$ is the storage porosity, $\Gamma$ is the counter-clockwise path along the boundary of the edge and $\phi_{\Gamma}$ is the conveyance porosity. $\mathbf{q}$ is the vector of conserved variables, $\mathbf{F}$ is the flux tensor and $\mathbf{n}$ is the unit normal vector, pointing outwards along $\Gamma . \mathbf{s}_{\Omega}$ and $\mathbf{s}_{\Gamma}$ are source term vectors. The vectors are defined as

$$
\mathbf{q}=\left[\begin{array}{c}
h \\
q_{x} \\
q_{y}
\end{array}\right], \quad \mathbf{F}=\left[\begin{array}{cc}
q_{x} & q_{y} \\
\frac{q_{x}^{2}}{h}+\frac{1}{2} g h^{2} & \frac{q_{x} q_{y}}{h} \\
\frac{q_{x} q_{y}}{h} & \frac{q_{y}^{2}}{h}+\frac{1}{2} g h^{2}
\end{array}\right], \quad \mathbf{s}_{\Omega}=\left[\begin{array}{c}
s_{m} \\
s_{f, x}+s_{d, x}+s_{z, x} \\
s_{f, y}+s_{d, y}+s_{z, y}
\end{array}\right], \mathbf{s}_{\Gamma}=\left[\begin{array}{c}
0 \\
\frac{1}{2} g h_{0} \\
\frac{1}{2} g h_{0}
\end{array}\right],
$$

with $h$ being the water depth, $q_{x}$ and $q_{y}$ being the unit discharges in $x$ - and $y$-direction, respectively, where $x$ and $y$ are two directions of the Cartesian coordinate system. $s_{m}$ is the mass source term, $s_{f, x}$ and $s_{f, y}$ are the friction source terms in $x$ - and $y$-direction, respectively, $s_{d, x}$ and $s_{d, y}$ are the building drag source terms in $x$ - and $y$-direction, respectively, $s_{z, x}$ and $s_{z, y}$ are the bottom slope source terms in $x$ - and $y$-direction, respectively, $g$ is the gravitational acceleration and $h_{0}$ is the water depth evaluated for a constant water elevation inside the cell.

The friction source term is calculated using Manning's formulation as

$$
s_{f, j}=-g n^{2} h^{-7 / 3} q_{j}\|\mathbf{q}\|, \quad j=x, y,
$$

where $n$ is Manning's coefficient. The building drag dissipation can be calculated using a drag dissipation formula, cf., e.g. [9, 13, and cited references therein]. In the present work, we have neglected the building drag source term to focus solely on the mesh convergence.

\section{Numerical methods}

We solve the integral porosity shallow water equations derived in [9] by means of a first order accurate Godunov-type finite volume discretization on unstructured triangular grids. Numerical 


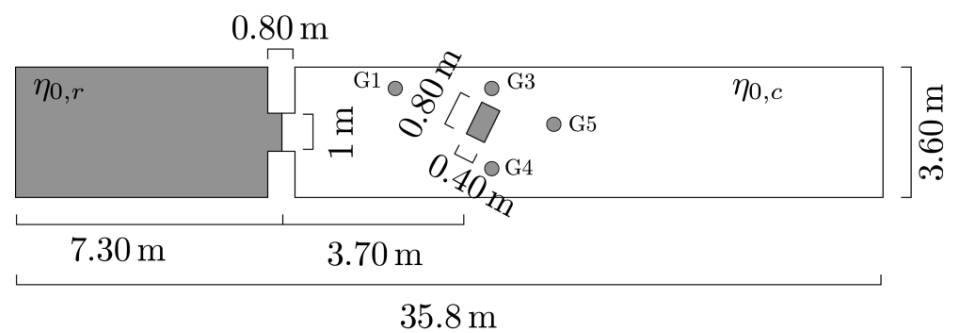

Figure 1: Geometry of the experiment and location of gauges

fluxes over the cell edges are calculated with a Harten, Lax and van Leer approximate Riemann solver with the contact wave restored (HLLC) [11]. The bottom slope source term is discretized using the divergence formulation presented in [12].

The friction source term is discretized in a point implicit way [2]. Time integration is carried out using an explicit forward Euler method.

\section{Mesh refinement techniques}

Meshes are generated using the mesh generator Gmsh [4]. We investigate three types of refinement strategies: (1) uniform mesh refinement (new cells are created by dividing existing cells) (UMR), (2) manual mesh refinement (meshes are created from scratch for each refinement stage) (MMR), (3) mesh refinement with local coarsening (cell size in regions around obstacles is kept constant during the refinement to keep the model at macroscale) (LCR).

\section{Case study}

The presented case study is a laboratory experiment conducted at the facilities of the Université catholique de Louvain, and a detailed discussion of the experimental setup, channel geometry and measurement techniques can be found in the corresponding article [10].

The experiment consists of a reservoir with an initial water depth of $\eta_{0, r}=0.4 \mathrm{~m}$, that is connected by a gate to a straight channel with a thin layer of $\eta_{0, c}=0.02 \mathrm{~m}$ of water. The gate is initially closed. A single obstacle with dimensions $0.4 \mathrm{~m}$ by $0.8 \mathrm{~m}$ is placed with an angle of $64^{\circ}$ to the $x$-axis inside the channel. The gate is opened rapidly to generate a dam-break flow against this obstacle. The geometry of the experiment and the location of measurement gauges is sketched in Fig. 1. The grey area indicates the reservoir, the white area is the channel. The Manning's coefficient in the domain is given as $n=0.01 \mathrm{~ms}^{-1 / 3}$.

For UMR and MMR, the cell size is chosen such that the cell size in the reservoir $l_{c, R}$ relates to the cell size in the channel $l_{c, C}$ as

$$
l_{c, R}=2.5 l_{c, C}
$$

and the cell size around the obstacle $l_{c, O}$ is set in relation to $l_{c, C}$ as

$$
l_{c, 0}=1.5 l_{c, C}
$$



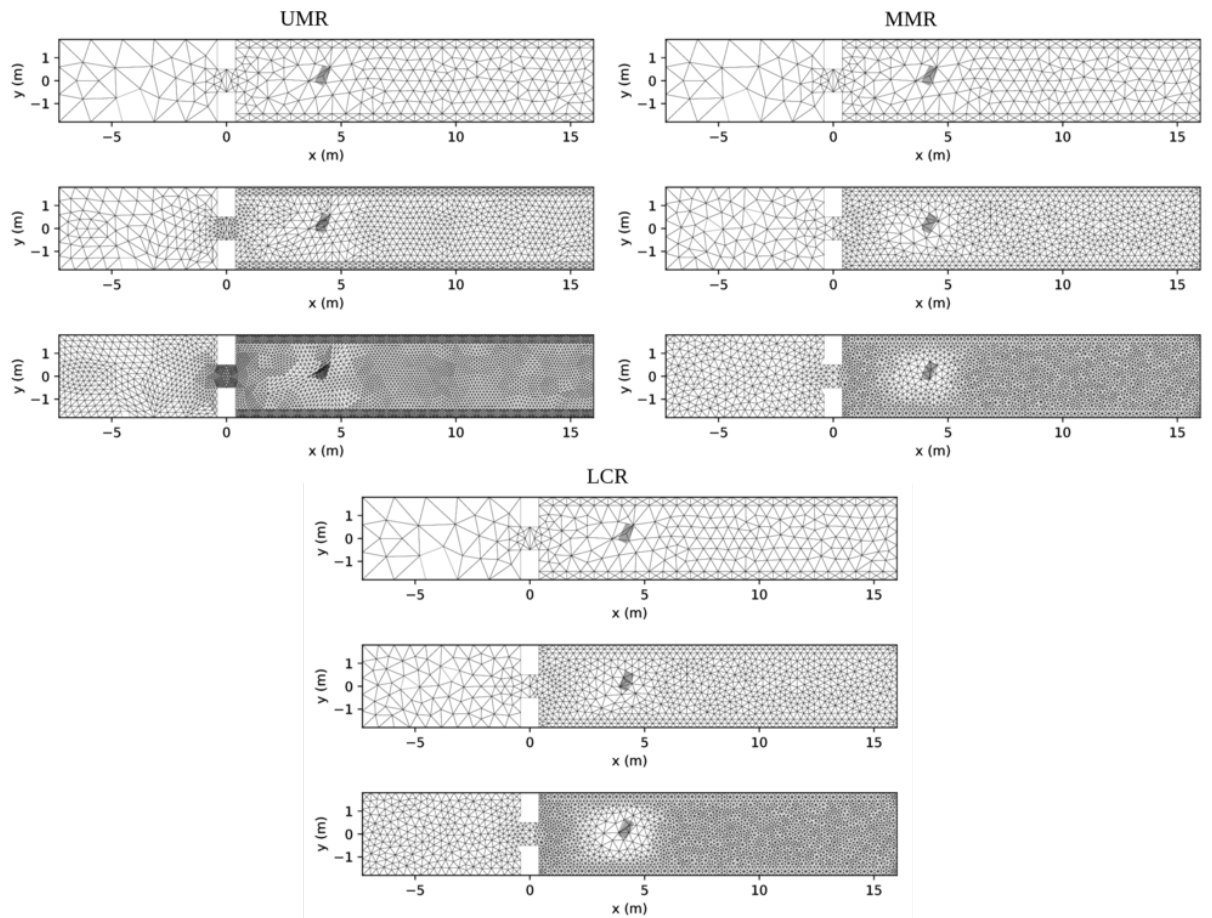

Figure 2: Meshes resulting from different refinement techniques for $l_{c, C}$-values of $0.6 \mathrm{~m}$ (top in each subfigure), $0.3 \mathrm{~m}$ (middle in each subfigure) and $0.15 \mathrm{~m}$ (bottom in each subfigure)

For LCR, Eq. 4 holds, but $l_{c, O}$ is held constant at $0.9 \mathrm{~m}$ for all refinement steps. $l_{c, C}$ is halved at each refinement step, i.e. from $0.6 \mathrm{~m}$ to $0.3 \mathrm{~m}$ and so on, down to $0.01875 \mathrm{~m}$. The meshes for $l_{c, C}$-values of $0.6 \mathrm{~m}, 0.3 \mathrm{~m}$ and $0.15 \mathrm{~m}$ for each refinement technique are shown in Fig. 2.

Forcing the center of the building polygon as a vertex into the triangulation is considered good practice for designing meshes for the IP model. It is seen in Fig. 2 that this leads to distorted cells. In UMR, this distortion is propagated to the finer meshes. In MMR and LCR, new distorted elements may occur.

The total number of cells resulting from different refinement techniques is shown in Fig. 3. This number indicates how similar two refined meshes are. If the total number of cells shows a linear increase if plotted on a log-axis, the refinement technique is said to yield similar meshes. As expected, for UMR the cell number increases proportional to the power of 4 in each refinement step, thus giving similar meshes that are ideal to use for mesh convergence studies. MMR and LCR yield less number of cells and show a similar trend. LCR yields the lowest number of cells, because the cells around the obstacle are kept large. Therefore, the MMR and LCR refinement yields meshes that are not necessarily similar and may not be optimal to study mesh convergence. 


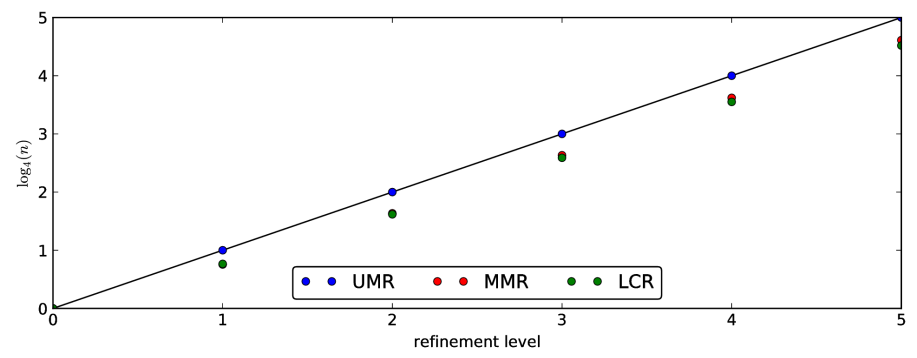

Figure 3: Total cell number resulting from different refinement techniques

\section{Results}

Results obtained with the three finest meshes are plotted in Fig. 4. We see that for all meshing strategies, results at gauge 1 start to oscillate at arount $t=15 \mathrm{~s}$. Comparison with snapshots from the simulation (not shown here) reveal that this coincides with the time that a backwards propagating hydraulic jump arrives at this point. Results at gauge 3 converge for all meshes. Gauge 4 gives mixed results, for UMR and MMR mesh convergence is achieved, but for LCR results still show no convergence.

At simulation snapshots, we observe a Kármán vortex emerging for $l_{c, C}=0.01875 \mathrm{~m}$. For illustration purposes, we provide a snapshot at $t=13 \mathrm{~s}$ for a very high-resolution simulation with $l_{c, C}=0.01 \mathrm{~m}$ in Fig. 5 . This explains the oscillations observed at gauge 5 for UMR and MMR. For LCR, the mesh in this region is not fine enough for the Kármán vortex to develop. Thus, model results converge at gauge 5 for LCR.

\section{Conclusions and outlook}

Grid convergence could be reached only at gauges 3 and 4, which are not directly impacted by the emerging Kármán vortices and the backwards propagating hydraulic jump. Model results at gauges 1 and 5 show oscillations when the cell size is small. We conclude that in cases where the obstacle density is low, high-frequency oscillations may appear in the domain, e.g. the periodic Kármán vortex shedding. These cannot be captured accurately by the integral porosity shallow water model, unless high resolutions similar to the conventional shallow water model are used. This violates the general principle and the purpose of using a macroscopic model. The benefit of using the integral porosity model at this point becomes similar to using an immersed boundary method, i.e. building boundaries do not need to be discretized explicitly. In this study, we presented ongoing research that examines the grid convergence property of the integral porosity shallow water model. We also plan to study cases where the flow is through a building array. We expect a different convergence behaviour, because multiple reflections inside the building array will hinder the development of the Kármán vortex. We will investigate whether the porosity model is capable of predicting the small scale fluctuations caused by the multiple reflections inside the building array. Furthermore, the influence of the building drag dissipation source term on the Kármán vortex shedding and small scale fluctuations will be investigated. 


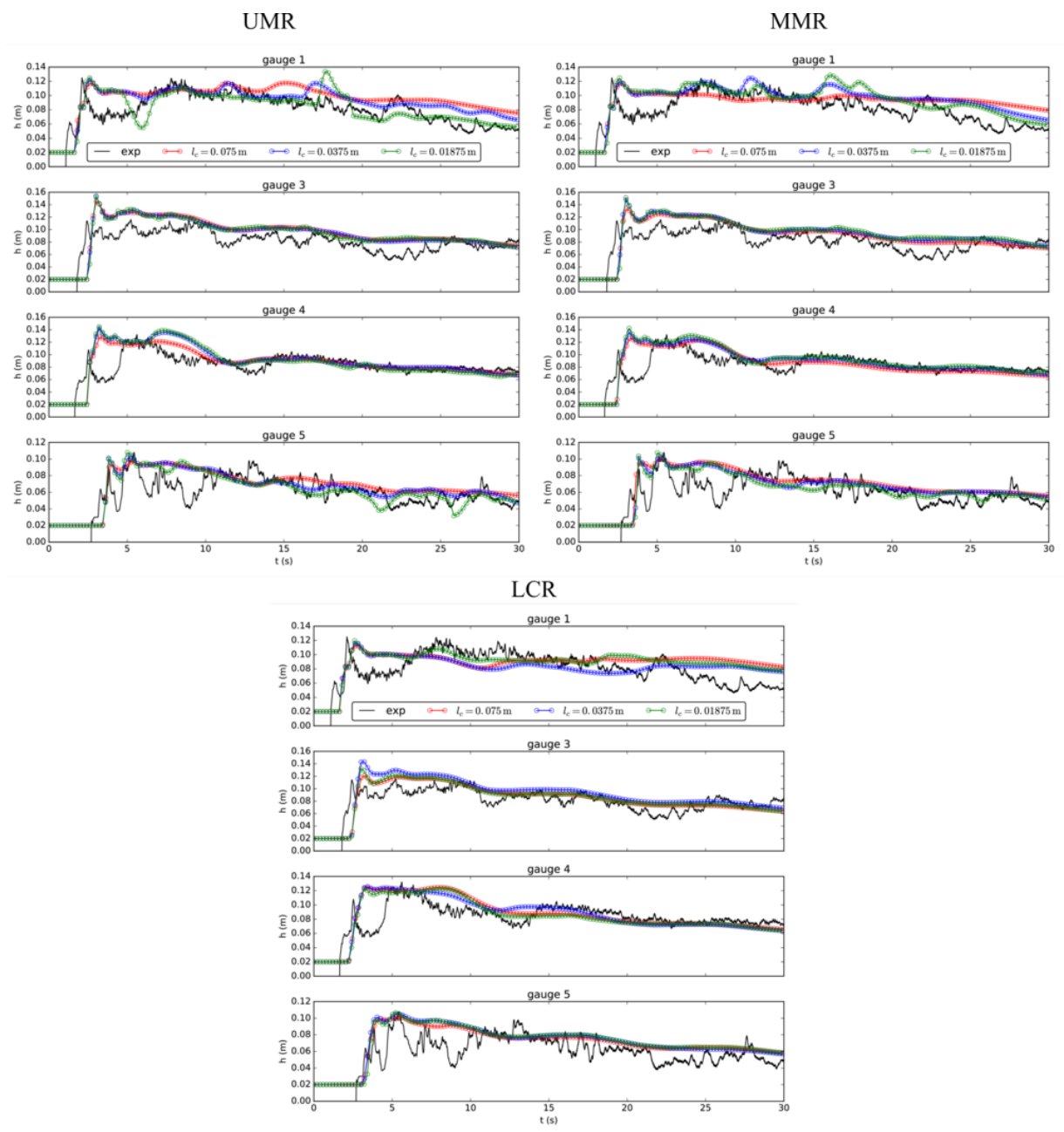

Figure 4: Model results using different meshes

\section{References}

[1] Martin Bruwier, Pierre Archambeau, Sébastien Erpicum, Michel Pirotton, and Benjamin Dewals. Shallow-water models with anisotropic porosity and merging for flood modelling on Cartesian grids. Journal of Hydrology, 554:693-709, 2017.

[2] Thomas R. A. Bussing and Earll M. Murman. Finite-volume method for the calculation of compressible chemically reacting flows. AIAA Journal, 26(9):1070-1078, sep 1988.

[3] Andrea Defina. Two-dimensional shallow flow equations for partially dry areas. Water Resources Research, 36(11):3251-3264, 2000.

[4] Christophe Geuzaine and Jean-François Remacle. Gmsh: A 3-d finite element mesh generator with built-in pre- and post-processing facilities. International Journal for Numerical Methods in Engineering, 79(11):1309-1331, 2009.

[5] Vincent Guinot. Multiple porosity shallow water models for macroscopic modelling of urban floods. 

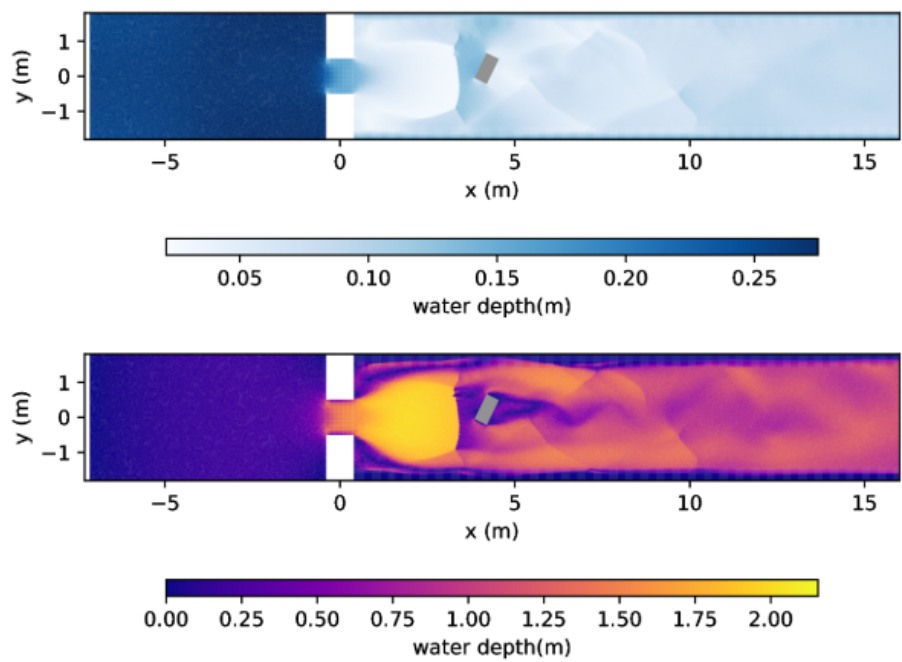

Figure 5: Snapshot at $t=13 \mathrm{~s}$ for water depth (top) and flow velocity (bottom), obtained with a high-resolution simulation

Advances in Water Resources, 37:40-72, 2012.

[6] Vincent Guinot, Brett F. Sanders, and Jochen E. Schubert. Dual integral porosity shallow water model for urban flood modelling. Advances in Water Resources, 103:16-31, 2017.

[7] Vincent Guinot and Sandra Soares-Frazão. Flux and source term discretization in two-dimensional shallow water models with porosity on unstructured grids. International Journal for Numerical Methods in Fluids, 50(3):309-345, 2006.

[8] Jean-Michel Hervouët, René Samie, and Benôit Moreau. Modelling urban areas in dam-break floodwave numerical simulations. In Proceedings of the International Seminar on Rescue Actions based on Dambreak Flow Analysis, Seinâjoki, Finland, 2000.

[9] Brett F. Sanders, Jochen E. Schubert, and Humberto A. Gallegos. Integral formulation of shallowwater equations with anisotropic porosity for urban flood modeling. Journal of Hydrology, 362(12):19-38, 2008.

[10] Sandra Soares-Frazão and Yves Zech. Experimental study of dam-break flow against an isolated obstacle. Journal of Hydraulic Research, 45(July 2015):27-36, 2007.

[11] Eleuterio F. Toro, M. Spruce, and W. Speares. Restoration of the contact surface in the HLLRiemann solver. Shock Waves, 4(1):25-34, 1994.

[12] Alessandro Valiani and Lorenzo Begnudelli. Divergence Form for Bed Slope Source Term in Shallow Water Equations. Journal of Hydraulic Engineering, 132(7):652-665, 2006.

[13] Mirjana Velickovic, Yves Zech, and Sandra Soares-Frazão. Steady-flow experiments in urban areas and anisotropic porosity model. Journal of Hydraulic Research, 55(1):85-100, 2017.

[14] Daniele Pietro Viero, Paolo Peruzzo, Luca Carniello, and Andrea Defina. Integrated mathematical modeling of hydrological and hydrodynamic response to rainfall events in rural lowland catchments. Water Resources Research, 50(7):5941-5957, jul 2014.

[15] Daniele Pietro Viero and Mohammad Valipour. Modeling anisotropy in free-surface overland and shallow inundation flows. Advances in Water Resources, 104:1-14, 2017. 